CGPG-01/1-1

\title{
Black hole entropy calculations based on symmetries
}

\author{
Olaf Dreyer円, Amit Ghosh and Jacek Wiśniewskif \\ Center for Gravitational Physics and Geometry, Department of Physics, \\ The Pennsylvania State University, University Park, PA 16802-6300, USA
}

\begin{abstract}
Symmetry based approaches to the black hole entropy problem have a number of attractive features; in particular they are very general and do not depend on the details of the quantization method. However we point out that, of the two available approaches, one faces conceptual problems (also emphasized by others), while the second contains certain technical flaws. We correct these errors and, within the new, improved scheme, calculate the entropy of 3-dimensional black holes. We find that, while the new symmetry vector fields are well-defined on the "stretched horizon," and lead to well-defined Hamiltonians satisfying the expected Lie algebra, they fail to admit a well-defined limit to the horizon. This suggests that, although the formal calculation can be carried out at the classical level, its real, conceptual origin probably lies in the quantum theory.
\end{abstract}

October 31, 2018

\footnotetext{
*dreyer@phys.psu.edu

†ghosh@gravity.phys.psu.edu

¥jacek@phys.psu.edu
} 


\section{Introduction}

A microscopic derivation of black hole entropy has been one of the greatest theoretical challenges for any candidate quantum theory of gravity. String theory in case of some extremal and near-extremal black holes [1] and canonical quantum gravity in case of general non-rotating black holes [2] have produced very interesting results in this direction.

As an alternative to both these approaches, a set of very attractive ideas was suggested by Strominger and Carlip [3, 4, 5] over the last few years. Motivated by some earlier works [6, 7, 8] on the relation between symmetries and Hamiltonians, these authors argued that states of a quantum black hole should belong to a multiplet of a representation of a suitable Lie algebra. Counting the number of states in the multiplet would then provide the black hole entropy. The Virasoro algebra has been proposed as a natural candidate for symmetries in this context.

An attractive feature of these alternative approaches is that they are not tied to the details of any specific model of quantum gravity. Even more strikingly, the central objects in this construction, namely the Virasoro algebra, central charge etc. appear already at the classical level through the Poisson bracket algebra. The Planck length in the expression of the entropy arises only from replacing Poisson brackets by appropriate quantum commutators. Being essentially classical, the scheme is quite robust and in principle applicable to black holes in any space-time dimension.

The first work [3] applies this idea to $2+1$ dimensions in the context of the BTZ black hole [9]. The symmetries, however, are taken from a previous analysis [7] which is tailored to asymptotic infinity rather than black hole horizon. Therefore, it is not apparent why these symmetries are relevant for the black hole in the space-time interior. For example, in asymptotically flat, 4-dimensional space-times, the symmetry group at (null) infinity is always the Bondi-Metzner-Sachs group, irrespective of the interior structure of the space-time. Thus, the results of [3] are equally applicable to a star that has similar asymptotic behavior as that of the black hole. Subsequently, Carlip improved on this idea significantly by making the symmetry analysis in the near-horizon region. Conceptually this approach is much more satisfactory in that the black hole geometry is now at the forefront. However, at the technical level, this work [5] appears to have some important limitations. The purpose of the present paper is to elucidate and discuss these technical problems in some detail, and then to present a consistent calculation which correctly implements the general ideas of [3, 5] by a careful treatment of all the relevant technical issues.

The organization of the paper is as follows. In section 2 we discuss the technical framework set up in [5] and point to the difficulties that arise in the implementation of the ideas mentioned above. In section 3 we investigate two different sets of symmetries. In 3.1 we consider symmetries that are defined intrinsically on the horizon 
and see if a central charge can be obtained. (To find these symmetries, as suggested in [5], we use the isolated horizon framework [10].) We find that the answer is in the negative. In 3.2, we then consider potential symmetry vector fields defined in a neighborhood of the horizon as in [5]. In 3.3, we find the corresponding Hamiltonians, and calculate the corresponding Poisson brackets. From these we read off the central charge. We conclude in 3.4 with a calculation of the entropy. The discussion of the last three sub-sections can be regarded as a careful reworking of the ideas introduced by Strominger and Carlip. We find that the entropy is indeed proportional to the area but the proportionality factor differs from the one of Bekenstein and Hawking by a factor of $\sqrt{2}$. Perhaps more importantly, although the Poisson brackets between the Hamiltonians are well-defined, the symmetry vector fields underlying this calculation fail to admit a well-defined limit to the horizon. These issues are discussed in section 4. Some technical details relevant to section 3.1 are given in the appendix.

For concreteness, we work in $2+1$ dimensions. However, the framework should admit a straightforward generalization to arbitrary space-time dimensions.

\section{Re-examination of the symmetry based calcula- tions in 3 dimensions}

In the standard conventions (with $8 G=1$ ), the line-element of BTZ black hole in the Eddington-Finkelstein coordinates is given by

$$
\begin{aligned}
& d s^{2}=-N^{2} d v^{2}+2 d v d r+r^{2}\left(d \phi+N^{\phi} d v\right)^{2}, \\
& N^{2}=-M+\frac{r^{2}}{\ell^{2}}+\frac{J^{2}}{4 r^{2}}, N^{\phi}=-\frac{J}{2 r^{2}} .
\end{aligned}
$$

Here, $J$ and $M$ are two real parameters and $\ell$ is related to the negative cosmological constant as $\Lambda \ell^{2}=-1$. The black hole has a Killing-horizon at $r=r_{+}$defined by $N^{2}\left(r_{+}\right)=0$, or

$$
r_{+}^{2}=\frac{1}{2} M \ell^{2}\left[1+\sqrt{1-\frac{J^{2}}{M^{2} \ell^{2}}}\right]^{1 / 2}, \quad|J| \leq M \ell .
$$

For the purpose of calculations it is convenient to introduce Newman-Penrose like basis in $2+1$ dimensions which has two null vectors $l^{a}$ and $n^{a}$ and a space-like vector $m^{a}$ (all real). They satisfy the relations

$$
l \cdot l=n \cdot n=l \cdot m=n \cdot m=0,-l \cdot n=m \cdot m=1 .
$$

The $2+1$ dimensional metric can be expressed in such a basis as $g_{a b}=-2 l_{(a} n_{b)}+m_{a} m_{b}$. The corresponding inverse metric is $g^{a b}=-2 l^{(a} n^{b)}+m^{a} m^{b}$. In the rest of the paper 
we will assume that we have chosen the triad $l, n$, and $m$ in such a way that the vectors $l$ and $m$ are tangent to the horizon at the horizon. For the metric (2.1) a convenient choice of the basis vector fields is

$$
l=\partial_{v}+\frac{1}{2} N^{2} \partial_{r}-N^{\phi} \partial_{\phi}, n=-\partial_{r}, m=\frac{1}{r} \partial_{\phi} .
$$

and the corresponding one-forms that span the dual-basis are

$$
l=-\frac{1}{2} N^{2} d v+d r, n=-d v, m=r N^{\phi} d v+r d \phi .
$$

The covariant derivatives of the one-forms, like $\nabla_{a} l_{b}$, can be expressed solely in terms of the one-forms and the so-called Newman-Penrose coefficients (See e.g. [12]; an exposition of the formalism in $2+1$ dimensions can be found in the appendix of [13])

$$
\begin{gathered}
\nabla_{a} l_{b}=-\epsilon n_{a} l_{b}+\tilde{\kappa} n_{a} m_{b}-\gamma l_{a} l_{b}+\tau l_{a} m_{b}+\alpha m_{a} l_{b}-\rho m_{a} m_{b} \\
\nabla_{a} n_{b}=\epsilon n_{a} n_{b}-\pi n_{a} m_{b}+\gamma l_{a} n_{b}-\nu l_{a} m_{b}-\alpha m_{a} n_{b}+\mu m_{a} m_{b} \\
\nabla_{a} m_{b}=\tilde{\kappa} n_{a} n_{b}-\pi n_{a} l_{b}+\tau l_{a} n_{b}-\nu l_{a} l_{b}-\rho m_{a} n_{b}+\mu m_{a} l_{b}
\end{gathered}
$$

where, for the metric (2.1) and the tetrad (2.4) the coefficients are given by

$$
\begin{gathered}
\epsilon=\frac{r}{\ell^{2}}-r\left(N^{\phi}\right)^{2}, \rho=-\frac{1}{2 r} N^{2}, \mu=-\frac{1}{r} \\
\alpha=\tau=\pi=N^{\phi} \\
\tilde{\kappa}=\nu=\gamma=0 .
\end{gathered}
$$

At the horizon $\epsilon\left(r_{+}\right)=\kappa$, where $\kappa$ is the surface gravity of the black hole.

With these preliminaries out of the way, let us now apply the general ideas of [5] to this 3-dimensional black hole. The BTZ space-time admits a global Killing vector

$$
\chi=\partial_{v}-\Omega \partial_{\phi}, \quad \Omega=N^{\phi}\left(r_{+}\right) .
$$

As in [5] we now define another vector field $\rho^{a}$ which is given by

$$
\nabla_{a} \chi^{2}=-2 \kappa \rho_{a}, \quad \rho^{a}=\frac{r}{r_{+}}\left(\partial_{v}+N^{2} \partial_{r}-N^{\phi} \partial_{\phi}\right) .
$$


It follows that $\chi \cdot \rho=0$ and $\mathcal{L}_{\chi} \rho^{a}=0$ everywhere. For convenience we express both vector fields $\chi, \rho$ in the Newman-Penrose basis up to order $\left(r-r_{+}\right)^{2}$ terms

$$
\begin{aligned}
& \chi^{a}=l^{a}+\left(r-r_{+}\right)\left(\kappa n^{a}+2 \Omega m^{a}\right)+\mathcal{O}\left(r-r_{+}\right)^{2}, \\
& \rho^{a}=\frac{r}{r_{+}} l^{a}-\left(r-r_{+}\right) \kappa n^{a}+\mathcal{O}\left(r-r_{+}\right)^{2} .
\end{aligned}
$$

Clearly, at the horizon $\chi \hat{=} \rho \hat{=} l$. Two other useful identities are $\nabla_{a} \rho_{b}=\nabla_{b} \rho_{a}$ and $\chi^{a} \nabla_{a} \chi_{b}=\kappa \rho_{b}$ which follow from the definition (2.9) of $\rho$ and the fact that $\chi$ is a Killing vector.

As in [5], the classical phase-space can be taken to be the space of solutions of Einstein's equations. Each space-time configuration which is a point in the phase-space contains an inner as well as an outer boundary. Moreover, all space-time configurations in the neighborhood of the inner boundary are BTZ-like. For this to achieve [5] uses a set of boundary conditions which insure that all space-times admit a Killing vector $\chi$ in a neighborhood of the inner boundary and posses the same "near-horizon geometry'. More precisely, it requires

$$
\chi^{a} \chi^{b} \delta g_{a b} \hat{=} 0, \quad \chi^{a} t^{b} \delta g_{a b} \hat{=} 0,
$$

where $t^{a}$ is any space-like vector tangent to the inner boundary $(t \cdot \chi=0)$. The hat over the equality sign here means that the above equation holds on the horizon. Clearly the vector field $\xi$ which preserves these boundary conditions (2.12) under diffeomorphisms has to be tangent to the horizon. Keeping the same notation as in [5] let us take the vector field to be

$$
\xi^{a}=T \chi^{a}+R \rho^{a}
$$

where $R$ and $T$ are arbitrary functions. By demanding that (2.13) preserves (2.12) under diffeomorphisms one puts restrictions on $R$ and $T$. These are derived in [5] (cf. eq $(4.8))$

$$
R=\frac{1}{\kappa} \frac{\chi^{2}}{\rho^{2}} D T, \quad D \equiv \chi^{a} \nabla_{a} .
$$

The vector field, satisfying (2.14), can then be said to generate symmetries in the precise sense of (2.12).

Let us now check the closure of the Lie-algebra of these vector fields. It is at this point that the analysis of [5] appears to be flawed. The errors arise at three levels:

a) As noted in [5] the requirement that the Lie bracket of symmetry vector fields should close imposes a new condition

$$
\mathcal{L}_{\rho} T \hat{=} 0 .
$$


In [5] this condition was imposed at the horizon. However, at the horizon $\rho^{a} \hat{=} \chi^{a} \hat{=} l$ and hence, (2.15) reads $D T \hat{=} 0$. Then the main steps in the calculations of [5] fail to go through. In particular, the central charge is expressed in terms of DT at the horizon and therefore vanishes identically. This in turn implies that the entropy also vanishes identically. While the restriction on $D T$ has been noted explicitly in [5], its (obvious) consequences on the value of the central change and entropy are overlooked. b) Furthermore, it is not sufficient to impose (2.15) only at the horizon; closure will fail unless it holds in a neighborhood.

c) Later, for explicit calculations, a specific function $T$ is chosen in [5] (cf. eq. (5.6)) . Unfortunately, this function does not satisfy the condition (2.15) which is required in the earlier part of the analysis in [5].

In other words, although the boundary conditions (2.12) and (2.15) are reasonable, the technical implementation of them, as presented in [5], is incorrect. In the next section we will propose an implementation of the boundary conditions that does not suffer from these problems.

\section{New Sets of Symmetries}

The purpose of this section is to present a systematic analysis which is free of the technical flaws discussed above. However, before embarking on this discussion, in section 3.1 we first investigate a separate issue. In Carlip's analysis, the symmetry vector fields are defined in a neighborhood of the horizon. From general, classical considerations, one might expect that it should be possible to focus just on the horizon structure and consider symmetry vector fields defined intrinsically on the horizon. We consider this possibility in Section 3.1 and show that in this case the central charge vanishes. Thus the Carlip-type analysis can not be carried out with symmetries defined intrinsically on the horizon. This result suggests that, although the analysis appears to be classical, the origin of the central charge - and hence entropy - can not be captured in a classical analysis. In the remainder of the section, we consider symmetry vector fields more closely related to those of [5] and improve on that analysis.

\subsection{Geometrical symmetries}

A new framework that is naturally suited for the analysis of symmetries on the horizon is now available - the so-called 'isolated horizons'. This is a notion that captures the minimum structure intrinsic to the horizon to describe an equilibrium state of a black hole. It allows, however, for matter and radiation in an arbitrary neighborhood of the black hole, as long as none crosses the horizon. As suggested in [5], this framework 
is well-suited for the Carlip approach to entropy. A comprehensive description of the isolated horizons framework is given in [10, 11]. Isolated horizons in $2+1$ dimensions are discussed in detail in [13].

It is natural to define symmetries as maps which preserve the basic horizon structure, by which we mean the induced metric and a class of null generators. More details are given in the Appendix. Here let us just state that a vector field which preserves that structure must be tangent to the horizon, i.e. of the form

$$
\xi^{a} \hat{=} A l^{a}+B m^{a}
$$

where the functions $A$ and $B$ are restricted to be

$$
\begin{aligned}
& A=C\left(v_{-}\right)+\text {const. } \cdot v \\
& B=\text { const. }
\end{aligned}
$$

The coordinates $v$ and $v_{-}$are defined by the relations $n=-\mathrm{d} v, m=\frac{1}{r_{+}} \frac{\partial}{\partial \phi}$, and $v_{ \pm}=v \mp \phi / \Omega$. It is easy to see that the algebra of these vector fields (3.16) closes. Now the boundary conditions at the isolated horizon induce a natural symplectic structure in the phase space, where the phase space consists of all possible space-time configurations which admit a fixed isolated horizon. The symplectic structure can be used to evaluate the Poisson brackets between any two phase space functionals.

It is not difficult to check that the vector field (3.16), is Hamiltonian. For details see the Appendix. The Poisson brackets of the corresponding Hamiltonians close on-shell

$$
\left\{H_{\xi_{1}}, H_{\xi_{2}}\right\} \hat{=} H_{\left[\xi_{1}, \xi_{2}\right]}
$$

Hence, the central charge is zero. This result is not quite unexpected since our analysis is entirely classical and typically the central charge arises from the failure of the classical symmetries to be represented in the quantized theory. This shows that, in general, for symmetries represented by smooth vector fields on the horizon, the ideas of [3, 5] do not go through. If one wishes to use smooth fields - as is most natural at least in the classical theory - the central charge can arise only from quantization and the analysis would be sensitive to the details of the quantum theory, such as the regularization scheme used, etc. If the original intent of the ideas of [3, 5] is to be preserved, one must consider symmetries represented by vector fields which do not admit smooth limits to the horizon; in a consistent treatment, the use of "stretched horizons" [5] is not optional but a necessity. Perhaps this is the price one has to pay to transform an essentially quantum analysis in the language of classical Hamiltonian theory.

Finally, note that any reasonable local definition of a horizon should lead to the above conclusions since we have made very weak assumptions in this sub-section. 


\subsection{Extended notion of symmetries}

Let us now return to the discussion of Section 2 and consider symmetry vector fields defined in a neighborhood of the horizon. Thus, we will now use the stronger set of conditions (2.12) which requires that the closure condition (2.15) be satisfied every-

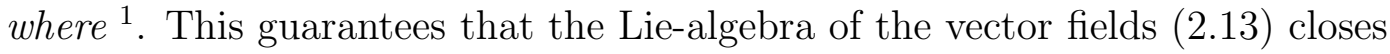

$$
\left[\xi_{T_{1}}, \xi_{T_{2}}\right]=\mathcal{L}_{\xi_{T_{1}}} \xi_{T_{2}}=\xi_{T_{1} D T_{2}-T_{2} D T_{1}}, \quad \xi_{T}=R \rho+T \chi
$$

where $R$ is determined in terms of $T$ as in (2.14). One is to make use of the facts that $\mathcal{L}_{\chi} \rho=\mathcal{L}_{\rho} T=\mathcal{L}_{\rho} R=0$. The condition (2.15), however, restricts the choice of the vector fields everywhere. To solve for the vector fields we consider a 'stretched'horizon at $r=r_{+}+\varepsilon$ as the inner boundary. The solutions that are of the form

$$
T_{n} \sim f_{n}(r) \exp \left(i n \Omega v_{+}\right)
$$

are especially interesting because they furnish a $\operatorname{Diff}\left(S^{1}\right)$, provided $f_{n} f_{m} \sim f_{n+m}$. However, the condition (2.15) is to be imposed carefully because of the $\left(r-r_{+}\right)$terms in the vector field $\rho(2.9)$

$$
\rho^{a} \nabla_{a} T \sim\left(\partial_{v_{+}}+N^{2} \partial_{r}\right) T=0 .
$$

Clearly, the radial derivative of $T$ blows up at the horizon. With the ansatz (3.21) there is a unique solution for $T_{n}$ in the neighborhood of the horizon

$$
T_{n}^{\varepsilon}=\frac{1}{2 \Omega} \exp \left(-i n \frac{\Omega}{\kappa} \log \left(r-r_{+}\right)+i n \Omega v_{+}\right) .
$$

The normalization of $T$ is so chosen that the vector fields $\xi$ form a $\operatorname{Diff}\left(S^{1}\right)$ algebra

$$
\left[\xi_{T_{n}}^{\varepsilon}, \xi_{T_{m}}^{\varepsilon}\right]=i(n-m) \xi_{T_{m+n}}^{\varepsilon}
$$

in the neighborhood of the horizon.

Notice that because of (3.23) the vector fields $\xi$ do not have a well defined limit at the horizon. They are defined only at the stretched horizon and oscillate wildly in the limit $r \rightarrow r_{+}$. Also the radial derivative of $\xi$ blows up, as expected from the condition (2.15). So one has to take great care in evaluating the Poisson bracket and Hamiltonians - now one cannot ignore terms which are of order $\mathcal{O}\left(r-r_{+}\right)$especially in presence of radial derivatives in the Poisson brackets. Actually more terms will contribute to the Poisson bracket and a thorough examination of the entire calculation is needed.

\footnotetext{
${ }^{1}$ Strictly speaking, we only consider a neighborhood of the horizon where the vector field $\chi$ is Killing. In the BTZ example, however, it is globally Killing
} 


\subsection{Hamiltonian and Poisson bracket algebra}

The existence of the Hamiltonian under the boundary conditions (2.12) is shown in [5]. The surface Hamiltonian is (the bulk Hamiltonian is zero by constraints)

$$
H_{\xi_{n}}^{\varepsilon}=\frac{1}{2 \pi} \oint_{S_{\Delta}} \epsilon_{a b c} \nabla^{b} \xi_{n}^{\varepsilon c} .
$$

The phase space, described in section 2 is associated with a conserved symplectic current [14]. The corresponding symplectic structure may be used to evaluate the Poisson brackets between any two functionals in the phase-space. On shell, the symplectic structure can be written as the sum of boundary terms only. However, one may choose appropriate fall-off conditions of the fields at asymptotic infinity such that the contribution from the outer boundary vanishes. In the present example the fields approach 'strongly' to asymptotic AdS-values. In that case given two Hamiltonian vector fields $\xi_{1}$ and $\xi_{2}$, the Poisson bracket between the two corresponding Hamiltonian functionals is given solely by the terms at the inner-boundary [14]

$$
\left\{H_{\xi_{1}}, H_{\xi_{2}}\right\}=\oint_{S_{\Delta}}\left(\xi_{2} \cdot \Theta\left[g, \mathcal{L}_{\xi_{1}} g\right]-\xi_{1} \cdot \Theta\left[g, \mathcal{L}_{\xi_{2}} g\right]-\xi_{2} \cdot\left(\xi_{1} \cdot L\right)\right)
$$

where $2 \pi \Theta_{a}[g, \delta g]=\epsilon_{a b}\left[g^{b c} \nabla_{c}\left(g_{d e} \delta g^{d e}\right)-\nabla_{c} \delta g^{b c}\right]$ is the one-form symplectic potential and $L$ is the three-form Lagrangian density. Making use of Einstein's equations $R_{a b}=2 \Lambda g_{a b}$ we can express the Poisson bracket explicitly in terms of the vector fields

$$
\left\{H_{\xi_{1}}, H_{\xi_{2}}\right\}=\frac{1}{2 \pi} \oint_{S_{\Delta}} \epsilon_{a b c}\left[\xi_{2}^{b} \nabla_{d}\left(\nabla^{d} \xi_{1}^{c}-\nabla^{c} \xi_{1}^{d}\right)+8 \Lambda \xi_{2}^{b} \xi_{1}^{c}-(1 \leftrightarrow 2)\right] .
$$

Our purpose is to find the terms proportional to $n^{3}$ in the Poisson bracket (3.27) which give rise to a non-trivial central extension to the Poisson bracket algebra. The Hamiltonian (3.25) contains terms only linear in $n$. The central charge can then be read off from the $n^{3}$ terms with appropriate normalizations. After a long calculation we arrive at the following expression

$$
\lim _{\varepsilon \rightarrow 0}\left[\left\{H_{\xi_{n}}^{\varepsilon}, H_{\xi_{m}}^{\varepsilon}\right\}\right]=4 i n^{3} \delta_{m+n} \frac{a_{\Delta} \Omega}{2 \pi \kappa}+\text { terms linear in } n .
$$

Notice that although the vector fields (3.23) do not have a smooth limit as $r \rightarrow r_{+}$ the Hamiltonian and the Poisson bracket have well defined limits.

\subsection{Entropy arguments}

According to the standard normalization (up to linear order terms in $n$ )

$$
\lim _{\varepsilon \rightarrow 0}\left[\left\{H_{\xi_{n}}^{\varepsilon}, H_{\xi_{m}}^{\varepsilon}\right\}-i(n-m) H_{\xi_{n+m}}^{\varepsilon}\right]=i \frac{c}{12} n^{3} \delta_{n+m}
$$


the central charge can be read off from the $n^{3}$-term in the Poisson bracket (3.27)

$$
c=24 \frac{a_{\Delta} \Omega}{\pi \kappa} .
$$

The zero mode of the Hamiltonian too can be read off from (3.25) and is given by

$$
\lim _{\varepsilon \rightarrow 0}\left[H_{\xi_{0}}^{\varepsilon}\right]=\frac{a_{\Delta} \kappa}{2 \pi \Omega} .
$$

Hence, by Cardy formula 15, the entropy is

$$
S=2 \pi \sqrt{\frac{c H_{\xi_{0}}}{6}}=2 \sqrt{2} a_{\Delta}
$$

which agrees with the Bekenstein-Hawking entropy (in units $8 G=\hbar=1$ ) up to a factor of $\sqrt{2}$.

It is worth noting here that Carlip's central extension (see formula 5.10 of [5]) and zero-th mode Hamiltonian have the same numerical factor as ours. Nevertheless, he argues that one should use a different, so called effective central extension, and obtains the right numerical factor for entropy. In our case this strategy fails since we have an extra factor of $\Omega / \kappa$ or its inverse in front of our expressions. It should be stressed, however, that this factor is rigidly fixed by the requirements that the symmetry algebra closes, that it gives a $\operatorname{Diff}\left(S^{1}\right)$, and that the symmetry vector fields are periodic in the coordinate $\phi$ with the period $2 \pi$. Moreover, following the

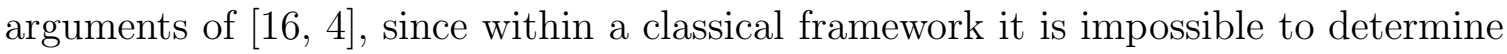
the value of the Hamiltonian in the ground state of the corresponding quantum theory, the right value of the central charge that is to be used in the Cardy formula is not determined classically.

\section{Discussion}

The entropy calculation of [3] faces certain conceptual limitations because the asymptotic symmetries may be completely different from the horizon symmetries. Both central charge (3.30) and Hamiltonian (3.25) are quite different from the ones found in [7] for asymptotic infinity. Thus, one needs an analysis restricted to the neighborhood of the horizon. In [5], Carlip recognized this limitation and carried out a Hamiltonian analysis using symmetries defined near the horizon. However, as we saw in section 2 , the resulting analysis has certain technical flaws. In particular, the vector fields which correctly incorporate the ideas laid out in the beginning of that paper are quite different from the ones used in the detailed analysis later on. 
In section 3 we made a proposal to overcome those technical problems and obtained a consistent formulation which implements the previous ideas. However, now the symmetry vector fields (2.13) do not have a well-defined limit at the horizon. Nonetheless both the Hamiltonians and their Poisson brackets are well-defined. Furthermore, there is a central charge which, following the reasoning of [3, 5], implies that the entropy is proportional to area. While the argument has attractive features, its significance is not entirely clear because the vector fields generating the relevant symmetries fail to admit well-defined limits to the horizon. Presumably, this awkward feature is an indication that, in a fully coherent and systematic treatment, the central charge would really be quantum mechanical in origin and could be sensitive to certain details of quantization, such as the regularization scheme used. Indeed, in the detailed analysis, we had to first evaluate the Poisson bracket and then take the $\operatorname{limit} \lim \epsilon \rightarrow 0$ (see expressions (3.31) and also (3.29) ), a step typical in quantum mechanical regularization schemes. Thus, it could well be that the awkwardness stems from the fact that, following [3, 5], we have attempted to give an essentially classical argument for a phenomenon that is inherently quantum mechanical.

This viewpoint is supported by our analysis of section 3.1 of symmetries corresponding to smooth vector fields. If one requires that vector fields generating symmetries be smooth at the horizon - a most natural condition in a fully classical setting we found that the central charge would be zero! Thus, the fact that the vector fields do not admit a smooth limit to the horizon is essential to the Carlip-type analysis. The fact that one has to 'push' the analysis an $\epsilon$ away from the horizon indicates that the procedure may be a 'short-cut' for a more complete quantum mechanical regularization [2.

This, however, raises some questions about the method in general: a) How satisfactory is the classical analysis and how seriously should one consider such vector fields? In particular, role of such vector fields in terms of space-time geometry is far from obvious since they are not even defined on the horizon. b) Why should this particular algebra be the focus of attention? c) Does the whole analysis suggest a

\footnotetext{
${ }^{2}$ Sometimes it is argued that only a classical central charge can give rise to the standard expression $a_{\Delta} / 4 G \hbar$ of entropy and a central charge induced by a quantum anomaly can only give corrections to this expression. This, however, need not be the case. The central charge of a truly quantum origin must be a dimensionless number and the only such possibility is $c \sim a_{\Delta} / G \hbar$. This would appear in the quantum Virasoro algebra as

$$
\left[\hat{L}_{n}, \hat{L}_{m}\right]=(n-m) \hat{L}_{n+m}+\frac{c}{12}\left(n^{3}-n\right) \delta_{m+n}
$$

where $\hat{L}_{n}$ 's are now quantum operators. The eigenvalue of $\hat{L}_{0}$ should also be dimensionless ( $\left.a_{\Delta} / G \hbar\right)$. Thus, the correct semiclassical expression of entropy can be reproduced even when the central charge comes from the quantum theory.
} 
rather transparent quantum mechanical regularization scheme and hence, systematically constrain the quantum theory?

The fact that our final expression of entropy differs from the standard HawkingBekenstein formula by a factor of $\sqrt{2}$ also provides a test for quantum gravity theories. The value $H_{\xi_{0}}$ appearing in Cardy's formula is of a quantum mechanical nature. A classical calculation may not give the right numerical value for it. It then follows that a quantum theory of gravity will give the correct value for the entropy provided it (a) has classical general relativity as its low energy limit, and, (b) the expectation value $H_{\xi_{0}}$ is $a_{\Delta} \kappa / 4 \pi \Omega$ (assuming $H_{\xi_{0}}$ is well defined in quantum theory).

In spite of the limitations of this calculation, the final result is of considerable interest because it is not a priori obvious that all the relevant subtleties of the full quantum mechanical analysis can be compressed in a classical calculation simply by stretching the physical horizon an $\epsilon$ distance away, performing all the Poisson brackets and then taking the limit $\epsilon \rightarrow 0$ in the final expressions. Note, however, that a careful treatment of technical issues that were overlooked in [0] was necessary to bring out these features. Indeed, our analysis provides the precise sense in which the original intention in [3, 5] of reducing the problem to a classical calculation is borne out in a technically consistent fashion.

\section{Appendix}

In this appendix we define what is called a weakly isolated horizon. It is a more general object then an isolated horizon, however it is sufficient for our purpose of finding the symmetries of the horizon.

Let $\Delta$ be a null hypersurface and $l$ a future pointing null normal vector field on $\Delta$. We will denote by $[l]$ the equivalence class of null normals which differ from $l$ only by a multiplicative constant. Let us also introduce a one-form $\omega_{a}$ defined intrinsically on $\Delta$ by:

$$
\nabla_{a \underline{a}} l_{b}=\omega_{a} l_{b} .
$$

The arrow in the above equation denotes the pull-back to $\Delta$.

We call a pair $(\Delta,[l])$ a weakly isolated horizon if and only if:

1. $\Delta$ is topologically $S^{1} \times \mathbf{R}$.

2. The expansion $\Theta_{(l)}$ of $l$ vanishes.

3. The equations of motion hold on $\Delta$. The stress-energy tensor $T_{a b}$ is such that $-T_{b}^{a} l^{b}$ is future directed and causal.

4. $\mathcal{L}_{l} \omega=0$, where $\omega$ is the one-form given by the equivalence class $[l]$. 
We will say that a vector field $\xi$ generates a symmetry of the horizon if the flow generated by $\xi$ on the phase-space preserves the basic structure of the horizon, namely $[l]$ and $q$. Here, $q_{a b} \equiv g_{q b}$. Thus we impose,

$$
\begin{gathered}
\mathcal{L}_{\xi} l \in[l], \\
\mathcal{L}_{\xi} q_{a b}=0 .
\end{gathered}
$$

It is not difficult to check that any vector field $\xi$ satisfying the above conditions can be written as

$$
\xi^{a}=A l^{a}+B m^{a},
$$

where $A=C\left(v_{-}\right)+$const. $\cdot v, B=$ const, and $v_{ \pm}, v$ are defined by the relations $n=-\mathrm{d} v, m=\frac{1}{r_{+}} \frac{\partial}{\partial \phi}$, and $v_{ \pm}=v \mp \frac{\phi}{\Omega}$. As in the main text we assume that the vector field $m^{a}$ is tangent to the horizon. Note that $C\left(v_{-}\right)$must be a periodic function, therefore one can perform a Fourier analysis and find a set of modes $\xi_{n}$.

Now, using Hamiltonian considerations, one can find the symplectic structure and Hamiltonians in the phase-space of isolated horizons. For details see [13]. The symplectic structure on-shell is equal to

$$
\Omega\left(\delta_{\xi}, \delta\right)=-\frac{1}{\pi} \oint_{S_{\Delta}}\left[\left(\xi \cdot A_{I}\right) \delta e^{I}+\left(\xi \cdot e^{I}\right) \delta A_{I}\right]+\tilde{\Omega}\left(\delta_{\xi}, \delta\right),
$$

where $\tilde{\Omega}$ is a gauge term which is not important for the present analysis. $A$ and $e$ are the connection one-form and the orthonormal triad, respectively. Using this expression one can find the Hamiltonian corresponding to $\xi$ as well as the Poisson bracket of two Hamiltonians. The corresponding expressions are

$$
\begin{aligned}
H_{\xi} & =-\frac{1}{\pi} \oint_{S_{\Delta}}\left(\xi \cdot A_{I}\right) e^{I}+C_{\Delta}, \\
\left\{H_{\xi_{1}}, H_{\xi_{2}}\right\} & =-\frac{1}{\pi} \oint_{S_{\Delta}}\left[\left(\xi_{1} \cdot A_{I}\right) \mathcal{L}_{\xi_{2}} e^{I}+\left(\xi_{1} \cdot e^{I}\right) \mathcal{L}_{\xi_{2}} A_{I}\right],
\end{aligned}
$$

where $C_{\Delta}$ is zero except when $\xi$ contains a constant multiple of $l$. Then we have $C_{\Delta}[c l]=c\left(M+2 r_{+} \kappa+J \Omega\right)$.

Subsequently, one can check that for any such symmetry vector fields

$$
\left\{H_{\xi_{1}}, H_{\xi_{2}}\right\} \hat{=} H_{\left[\xi_{1}, \xi_{2}\right]},
$$

and therefore there is no central extension of the corresponding algebra of conserved charges. 


\section{Acknowledgments}

We gratefully thank Abhay Ashtekar for discussions and various important suggestions. Also, we would like to thank Steve Carlip for valuable correspondence. The work of AG was supported by the National Science Foundation grant PHY95-14240 and the Eberly Research Funds of Penn State.

\section{References}

[1] A. Strominger and C. Vafa, Phys. Lett. B379 (1996) 99; J. Maldacena, PhD Thesis, hep-th/9607235.

[2] A. Ashtekar, J. Baez, A. Corichi and K. Krasnov, Phys. Rev. Lett. 80 (1998) 904; A. Ashtekar, J. Baez and K. Krasnov, gr-qc/0005126.

[3] A. Strominger, JHEP 9802 (1998) 009.

[4] S. Carlip, Phys. Rev. Lett. 82 (1999) 2828.

[5] S. Carlip, Class. Quant. Grav. 16 (1999) 3327.

[6] C. Teitelboim, Ann. Phys. (NY) 79 (1973) 542; 207; M. Henneaux and C. Teitelboim, Comm. Math. Phys. 98 (1985) 391.

[7] J. Brown and M. Henneaux, Comm. Math. Phys. 104 (1986).

[8] T. Regge and C. Teitelboim, Ann. Phys. 88 (1974) 286.

[9] M. Bañados, C. Teitelboim and J. Zanelli, Phys. Rev. D 48 (1993) 1506.

[10] A. Ashtekar, C. Beetle, O. Dreyer, S. Fairhurst, B. Krishnan, J. Lewandowski and J. Wiśniewski, Phys. Rev. Letters 85 (2000) 3564.

[11] A. Ashtekar, S. Fairhurst and B. Krishnan, Phys. Rev. D 62 (2000) 104025.

[12] J. Stewart, Advanced general relativity, Cambridge University Press 1991

[13] A. Ashtekar, O. Dreyer and J. Wiśniewski, Isolated Horizons in 2+1 Dimensions, in preparation.

[14] V. Iyer and R. M. Wald, Phys. Rev. D 50 (1994) 846.

[15] J. L. Cardy, Nucl. Phys. B 270 (1986) 186.

[16] D. Kutasov, N. Seiberg, Nucl. Phys. B 358 (1991) 600. 